Chapman University

Chapman University Digital Commons

Physical Therapy Faculty Articles and Research

Physical Therapy

$10-22-2019$

\title{
Multi-day Longitudinal Assessment of Physical Activity and Sleep Behavior Among Healthy Young and Older Adults Using Wearable Sensors
}

Rahul Soangra

Chapman University, soangra@chapman.edu

Follow this and additional works at: https://digitalcommons.chapman.edu/pt_articles

Part of the Other Rehabilitation and Therapy Commons, and the Physical Therapy Commons

\section{Recommended Citation}

Soangra R. Multi-day longitudinal assessment of physical activity and sleep behavior among healthy young and older adults using wearable sensors. IRBM. 2020;41(2):80-87. https://doi.org/10.1016/ j.irbm.2019.10.002

This Article is brought to you for free and open access by the Physical Therapy at Chapman University Digital Commons. It has been accepted for inclusion in Physical Therapy Faculty Articles and Research by an authorized administrator of Chapman University Digital Commons. For more information, please contact laughtin@chapman.edu. 


\section{Multi-day Longitudinal Assessment of Physical Activity and Sleep Behavior Among Healthy Young and Older Adults Using Wearable Sensors}

\section{Comments}

NOTICE: this is the author's version of a work that was accepted for publication in IRBM. Changes resulting from the publishing process, such as peer review, editing, corrections, structural formatting, and other quality control mechanisms may not be reflected in this document. Changes may have been made to this work since it was submitted for publication. A definitive version was subsequently published in IRBM, volume 41, issue 2, in 2019. https://doi.org/10.1016/j.irbm.2019.10.002

The Creative Commons license below applies only to this version of the article.

\section{Creative Commons License}

\section{(c) $($ †) $\Theta$}

This work is licensed under a Creative Commons Attribution-Noncommercial-No Derivative Works 4.0 License.

\section{Copyright}

Elsevier 


\section{Highlights}

- Aging affects activities of daily living in human beings.

- Low frequency movements are performed among young and older adults

- Healthy young adults produced significantly more movements at $1.1 \mathrm{~Hz}$

- At night, young adults produced significantly larger accelerations during transitions.

- Clinically, movement analysis can serve as tool for health diagnosis, and to monitor the effects of intervention treatment or disease progression. 
Objectives: The number of elderly people is growing rapidly and aging is found to affect activities of daily living in older adults. Older adults have been reported to perform less physical activity when compared to younger counterparts. In the perspective of movement behavior, there is paucity of knowledge how are elderly different from younger ones. It is not known whether they produce only low frequency movement accelerations or the overall number of movements produced are reduced in elderly. It is also not known how elderly and younger ones perform movement transitions throughout the duration of a day and during night-time sleep.

Material and methods: In this study, 10 healthy young and 10 healthy older adults wore an inertial measurement unit at their lower back for 3-days and performed regular daily living activities. The 24-hours of day was divided into four 6-hour time zones and transitions made by young and elderly were investigated. All participants performed their regular daily activities unhindered and longitudinal multi-day signals for acceleration and angular velocity were analyzed. Time-frequency analysis was performed using wavelet transform and frequency content of each movement performed was computed.

Results: We found that both young and older adults performed significantly more low amplitude movements than medium and high amplitude movements. Healthy young adults produced significantly more movements at the frequency of $1.1 \mathrm{~Hz}$ than older adults. Healthy young adults were also found to have produced significantly smaller number of transitions in the mid-phases of sleep. They were also found to produce significantly larger accelerations during night-time sleep transitions compared to their older counterparts.

Conclusion: The advantages of collecting multiple day longitudinal human movement data and sleep transition data can lead us to important clinical diagnosis. The information from longitudinal assessment can help develop lifestyle interventions for disease prevention, monitoring of chronic diseases to prevent or slow disease progression among elderly people. 


\section{Graphical Abstract}

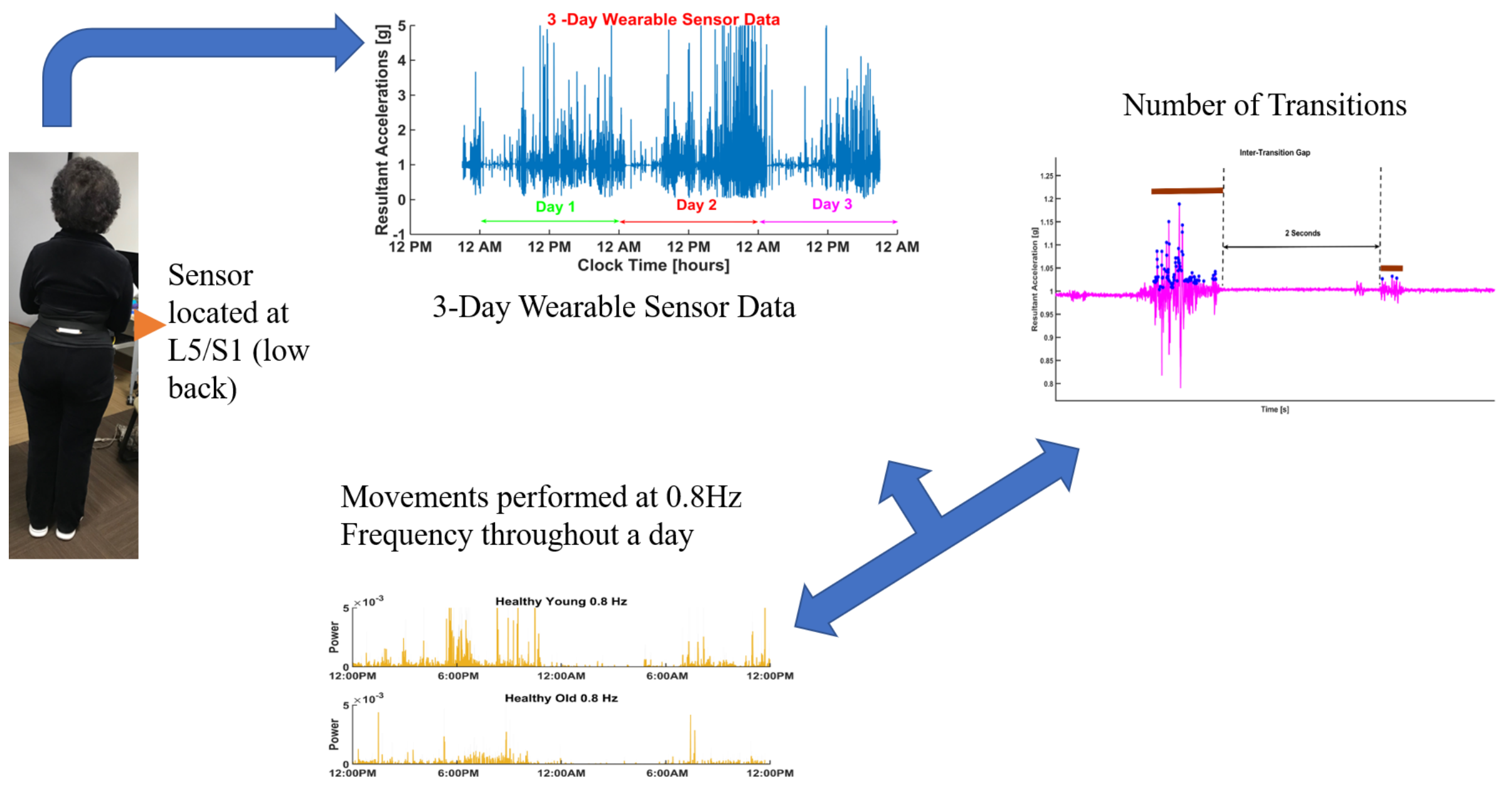

Multi-day Longitudinal Assessment of Movement and frequency of movement in Young and Older Adults 
Multi-day Longitudinal Assessment of Physical Activity and Sleep Behavior among healthy young and older adults using Wearable Sensors

Corresponding Author;

Rahul Soangra, PhD (soangra@chapman.edu)

Assistant Professor ,

Crean College of Health and Behavioral Sciences

Chapman University

9401 Jeronimo Rd, Room 229

Irvine, CA 92618

USA 


\begin{abstract}
The number of elderly people is growing rapidly and aging is found to affect activities of daily living. Older adults are found to perform less physical activity when compared to younger ones. In the perspective of movement behavior, it is not well understood how are elderly different from younger ones. It is not known whether they produce only low frequency movement accelerations or the overall number of movements produced are reduced in elderly. It is also not known how elderly and younger ones perform movement transitions throughout the duration of a day and during night-time sleep. In this study, 10 healthy young and 10 healthy old participants wore inertial measurement unit at their lower back for 3-days. The 24 hours of day was divided in to four 6 hour time zones and transitions made by young and elderly were investigated. All participants performed their regular daily activities unhindered and longitudinal multi-day signals for acceleration and angular velocity were analyzed. Time-frequency analysis was performed using wavelet transform and frequency content of each movement performed was computed. We found that both young and older adults performed significantly more low amplitude movements than medium and high amplitude movements. Healthy young adults produced significantly more movements at $1.1 \mathrm{~Hz}$ than older adults. Healthy young adults were also found to have produced significantly smaller number of transitions in the mid-phases of sleep. They were also found to produce significantly larger accelerations during night-time sleep transitions than their older counterparts. The advantages of collecting longitudinal data about human movement and sleep transition data can lead us to important clinical diagnosis. The information from longitudinal assessment can help develop lifestyle interventions for disease prevention, monitoring of chronic diseases to prevent or slow disease progression among elderly people.
\end{abstract}

Keywords: wearable sensors; longitudinal monitoring; Activities of Daily Living; inertial measurement units.

\title{
Introduction:
}

Older people above the age of 65 years account for $14.9 \%$ (47.8 million) of the total population in the US(Bureau, 2018) and it is expected to rise up to 98.2 million by the year 2060(Bureau, 2018). Elderly people spend most of their time at home and maintaining an active lifestyle is crucial for sustaining their health. Although aging is associated with decreased mobility, balance, agility, strength and reduced sensory acuity but to stay fit one does not require strenuous physical activity, in fact, it is possible to stay physically active by keeping up with causal daily activities. Physical activity in the older population certainly offers good health and prolongs functional independence. Certain amount of physical activity is important to maintain physical and mental health(Centers for Disease Control and Prevention, 2015). Increasing activities of daily living (ADL) have potential to improve mental health, decrease risk of falls, strengthen muscles and bones, reduce risk of certain conditions like cardiovascular diseases and thereby improve overall motor function and reduce healthcare expenditure and in a whole increase life expectancy 
(Centers for Disease Control and Prevention, 2015). Physical activity like walking is undoubtedly an efficient way to perform exercise in older adults. Traditionally ADL are assessed using subjective scoring for example i) Stanford Health Assessment Questionnaire(Bruce \& Fries, 2003) ii) Physical Activity Scale for Elderly (PASE) (Logan, Gottlieb, Maitland, Meegan, \& Spriet, 2013). These subjective scale reporting suffer from biases and errors related to cognitive impairment of the patients (Carlsson, Haak, Nygren, \& Iwarsson, 2012). Nowadays, wearable motion sensors have emerged as an objective alternative for monitoring human movement associated with ADL among older adults(Liu, Sohn, \& Kim, 2017). There is a growing number of population which is health conscious and uses wearable motion sensor embedded devices like smart watches to track number of steps or walking time. These devices are helpful in self-monitoring, goal attainment and adherence to the habit of walking. Undoubtedly, wearable sensors offer many advantages such as being light weighted, cost-effective, unhindered and portable data collection, and thus make them suitable for multiday longitudinal assessment in daily living environments (Allet, Knols, Shirato, \& de Bruin, 2010; Gokalp \& Clarke, 2013; Mathie, Celler, Lovell, \& Coster, 2004). One of the applications of wearable sensors is to longitudinally track human movement throughout the day, which can provide objective indication of health status and movement profile of a subject. Longitudinal tracking can provide important clinical information about frequency of movements throughout day, number of transitions made, duration and magnitude of each transition. These motion signals can also provide information about the sleep-related movement behavior by number of transitions and transition duration during the night. This information is clinically meaningful not only in evaluating the level of functionality of elderly, but also as an objective measure of quality of life or health status through movement quantification in older adults. The important relationship between sleep and activities of daily living is well known (Driscoll et al., 2008; Potter et al., 2016). Older adults have been found to have sleep problems such as premature awakening, fragmented sleep patterns, and reduced depth of sleep (Czeisler et al., 1992). Researchers have used wearable sensors to understand sleep disorders in patients with chronic respiratory disease and have validated these sensors against night vision video analysis during nighttime for assessing different postures and transitions (Gloeckl et al., 2015). They found wearable activity monitor with high degree of sensitivity and specificity to detect different nocturnal postures as well as large and medium sized transitions in patients with COPD (Gloeckl et al., 2015). To our knowledge, currently there exists no knowledge on how longitudinal motion tracking can provide clinically relevant information. The objective of this study is to explore new methods to quantify movement for longitudinal multi-day wearable sensor motion data. The findings from this study would contribute to the understanding of amplitude and frequency of the movements among healthy young and older subjects.

\section{Materials and Methods}

Participants: Twenty participants were recruited for this study. Ten healthy older adults (above 65 years of age) and 10 young adults were included in this study. The older subjects were community dwelling seniors who participate in community exercises conducted by Chapman 
University. The body-fixed sensor or wearable sensor used is Dynaport (Motion Monitor+ McRoberts BV, The Hague, The Netherlands), which is small, lightweighted, easily wearable and highly transportable. The Dynaport contains three axis piezo-capacitive acceleration and gyroscope sensors, each measuring at a sample rate of $100 \mathrm{~Hz}$. Data is stored on an inbuilt SD card. The sensor (size $84 \mathrm{~mm} \mathrm{X} \mathrm{50mm} \mathrm{X} 8 \mathrm{~mm}$; weight $44.5 \mathrm{~g}$ ) is placed in a belt which is strapped around the waist. It is positioned at $\mathrm{L} 5 / \mathrm{S} 1$, above posterior iliac spine. We chose low back as a location for sensor attachment, since low back has been reported as a suitable position for gait related movement assessments (Brandes, Zijlstra, Heikens, van Lummel, \& Rosenbaum, 2006; Zijlstra, 2004; Zijlstra \& Hof, 2003). The attachment of sensor at lower back has limited interference with daily activity and requires low power(Aminian \& Najafi, 2004), which makes it useful for longitudinal assessments (up to 7 consecutive days). All data processing algorithms utilizing the resultant acceleration and gyroscopic signals were implemented in MATLAB R2018a (The Mathworks Inc., USA). The exclusion criteria for this study were any kind of mobility impairments or disease for example orthopedic, neurological etc. that could affect gait, and ADL of the participants. All subjects gave written informed consent prior to the study. The protocol was approved by Chapman University IRB and California State University IRB.

Although the general principal of movement classification is to detect body postures, phases between static and dynamic movements(Lockhart, Soangra, Zhang, \& Wu, 2013; Soangra \& Lockhart, 2012; Soangra, Lockhart, Lach, \& Abdel-Rahman, 2013). In this study, we are proposing simple threshold-based algorithms which easily detect and quantify movements in longitudinal multi-day inertial sensor data.

Free-living Activity Performance and Measurements: Measuring the domains of activity behavior over the 24-h day (in multi-day assessment) is not only limited to specific activities like sit-to-stand, sit-to walk etc..., that can be measured in a laboratory but is dependent on measuring free-living activities performed unhindered in-home environments. In this study, all ADL's and sleep measurements were recorded using a single waist worn wearable sensor. We have previously reported a single inertial sensor is sufficient for detection and quantification of postural transitions and activities of daily living (Lockhart et al., 2013; Soangra \& Lockhart, 2012). All signal processing algorithm in this study were developed to understand human movement, transitions and frequency of movement in healthy young and older individuals. Each small movement during the day-time or night-time can be broken down into smaller units of 'transition' or 'burst of signal activity'. Movement Analysis: Resultant acceleration (R_XYZ) was defined as resultant acceleration from all three unidirectional accelerometers.

$$
R_{-} X Y Z=\sqrt{ }\left(\left(\llbracket A c c X \rrbracket^{\wedge} 2+\llbracket A c c Y \rrbracket^{\wedge} 2+\llbracket A c c Z \rrbracket^{\wedge} 2\right)\right)
$$

Activities of Daily Living: We computed detrended resultant acceleration (DRA) signals for analysis of magnitudes of activities of daily living.

$$
\text { Detrended Resultant Acceleration }(D R A)=\left|R_{A, x y z}-g\right|
$$

Where $\mathrm{g}$ is acceleration due to gravity. A one-second moving window size was chosen, since activities like sit-to-stand take about 2.2 seconds on average (Soangra \& Lockhart, 2012). Through pilot testing we found out that breathing and slight postural movements/adjustments 
such as head rotation and slight trunk movements produce mean DRA less than $20 \%$ of $g$ (gravity) and the standard deviations of less than $2 \%(<0.02 \mathrm{~g})$. We also collected pilot data for walking trial signals from healthy young and older subjects and found normal walking DRA signals were below $50 \%$ of $g$ and standard deviation less than $20 \%$ of $g$. Thus, we have categorized movement signals into three categories: low, medium and high amplitude movements (table 1). Where, m_DRA stands for mean of 1-second DRA data and std_DRA stands for standard deviation of 1-second DRA data.

Table 1: Categorization of activity into low, medium and high level activity using mean and standard deviation from DRA signals.

\begin{tabular}{|c|c|c|c|c|c|}
\hline \multicolumn{2}{|c|}{ Low } & \multicolumn{2}{|c|}{ Medium } & \multicolumn{2}{|c|}{ High } \\
\hline Mean & $\begin{array}{c}\text { Standard } \\
\text { Deviation }\end{array}$ & Mean & $\begin{array}{c}\text { Standard } \\
\text { Deviation }\end{array}$ & Mean & $\begin{array}{c}\text { Standard } \\
\text { Deviation }\end{array}$ \\
\hline $\begin{array}{l}\text { m_DRA }<0 . \\
2 \mathrm{~g}\end{array}$ & $\begin{array}{l}\text { std_DRA }<0.0 \\
2 \mathrm{~g}\end{array}$ & $\begin{array}{l}0.2 \\
\mathrm{~g}<\mathrm{m} \_\mathrm{DRA}<0 . \\
5 \mathrm{~g}\end{array}$ & $\begin{array}{l}0.02 \\
\mathrm{~g}<\text { std_DRA }<0 . \\
2 \mathrm{~g}\end{array}$ & $\begin{array}{l}\mathrm{m}_{5 \mathrm{~g}} \mathrm{DRA}>0 . \\
\end{array}$ & $\begin{array}{l}\text { std_DRA }>0 \\
2 \mathrm{~g}\end{array}$ \\
\hline
\end{tabular}

Transition: Each transition can be defined as burst of signal activity (figure 1). Utilizing movement data (R_XYZ), a moving window of 1-second will be used to evaluate all maxima's above the moving average by a threshold of $0.02 \mathrm{~g}$. There are several local maxima's (peaks) in one transition. The start and stop boundaries of these transitions are evaluated as below.

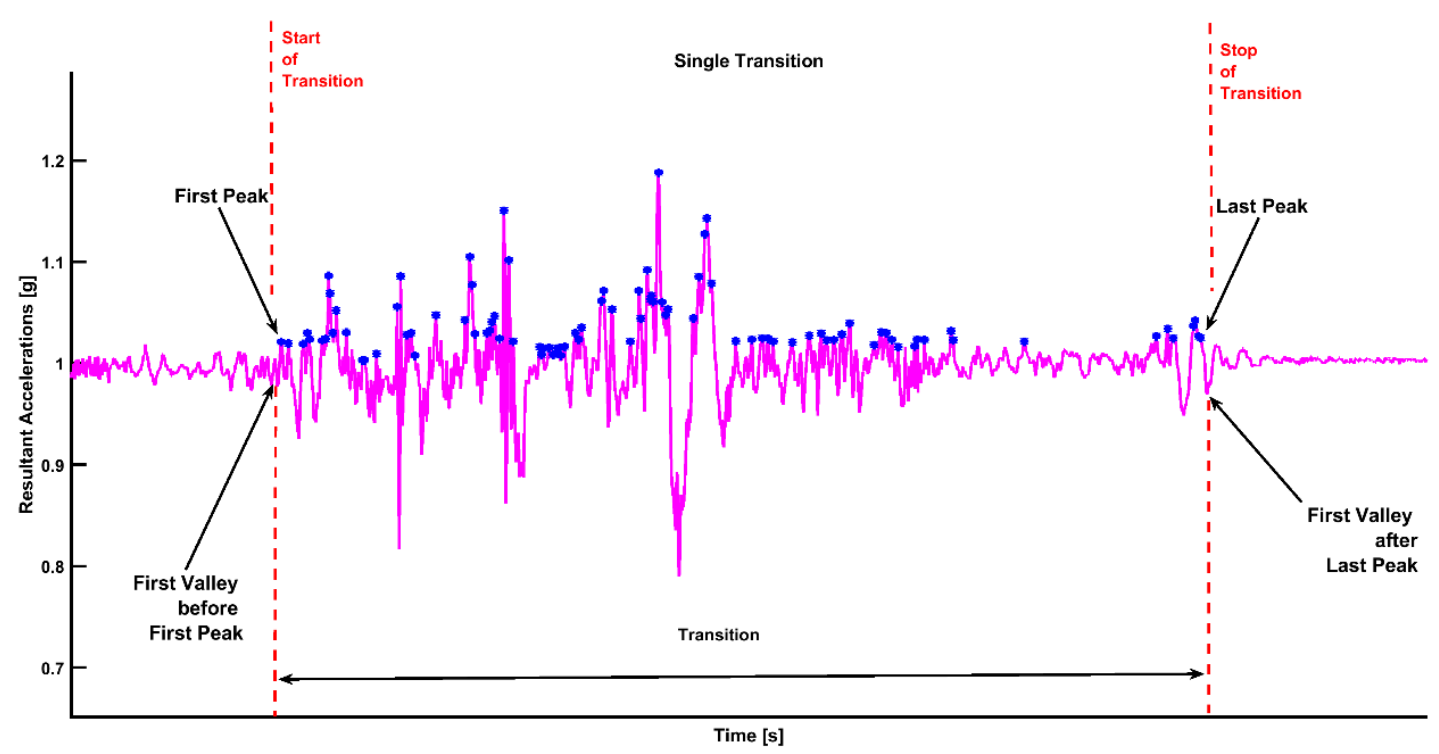

Figure 1: Start and stop of a transition signal

Start and Stop of a transition: The start point of a transition is defined as the first minima within an interval of $0.5 \mathrm{~s}$ before the first peak. Similarly, the stop point of a transition is defined as the first minima which occurs within $0.5 \mathrm{~s}$ after the last peak. Transition Duration: It is defined as the time interval from start of transition to stop of transition. Maximum and Minimum acceleration 
values in Transition: Maximum acceleration is the maximum acceleration (maximum peak value) within a transition duration. Minimum acceleration is the minimum acceleration (minimum valley value) during a transition.

Categorization of Transition: Any human movement produced may consist of a single or multiple signal transition. Each group of movement transitions (bundle of local maxima) are differentiated using a simple algorithm such as, if the time duration between local maxima is less than $2 \mathrm{~s}$, it is considered within the same transition. On the contrary if the time duration between two consecutive local maxima is more than $2 \mathrm{~s}$ - the local maxima's (peaks) are considered to be in separate transitions (Figure 2).

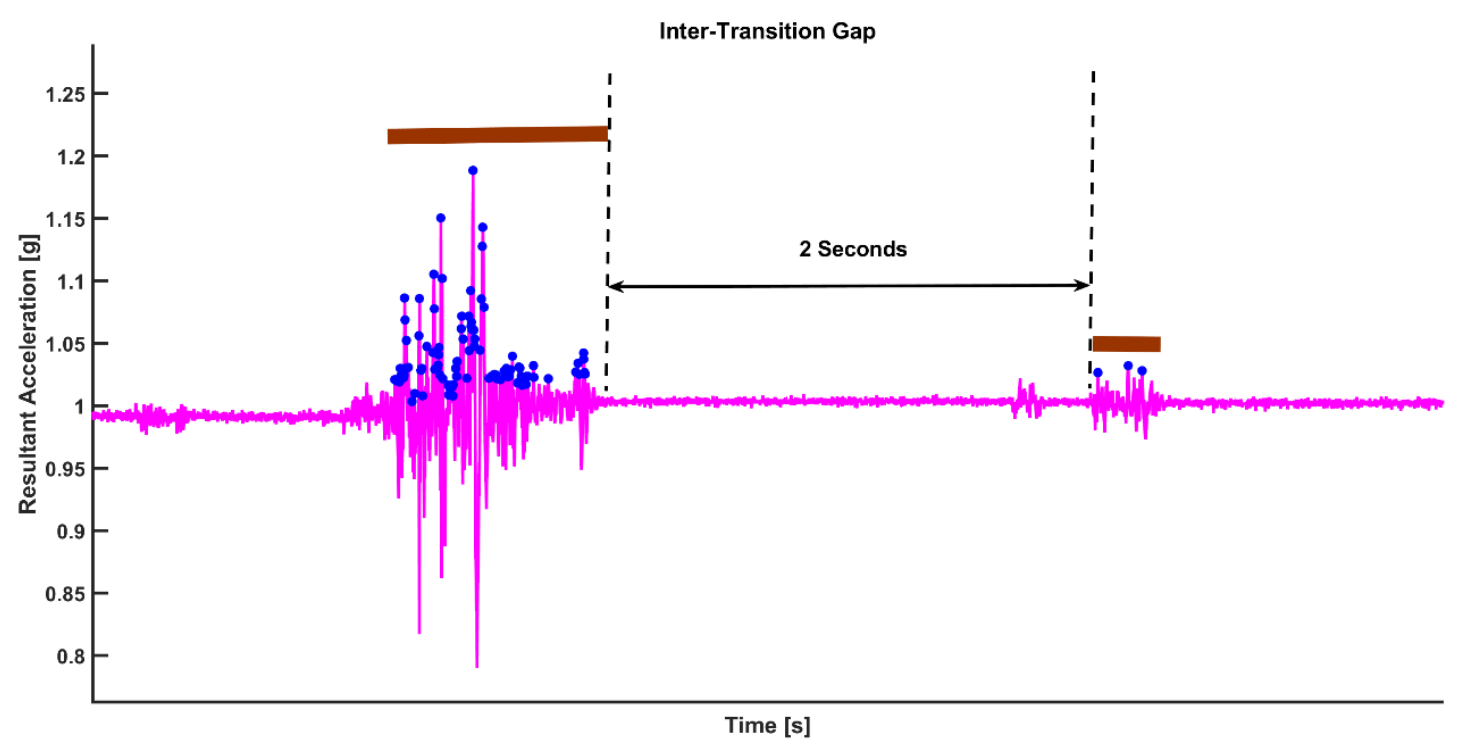

Figure 2: Inter-transition interval and transition durations in a transition

If two peaks are at least $2 \mathrm{~s}$ away in time from each other, we treat them as if two different movements occurred or different transitions. On the contrary, if two peaks are less than $2 \mathrm{~s}(<2 \mathrm{~s})$ away in time from each other, we treat as if they are part of same transition.

Sleep identification from 3-day longitudinal motion sensor data: Since all subjects wore the sensor on their low back, the accelerations were only produced in 2-D plane ( $\mathrm{X}$ and $\mathrm{Z}$ here) during lying down on bed (figure 3a). Resultant acceleration in XZ plane (denoted as $\mathrm{R}_{\mathrm{A}, \mathrm{xz}}$ ) of the sensor which encounter accelerations due to gravity while sleeping.

$$
R_{A, x z}=\sqrt{\left(A_{x}^{2}+A_{z}^{2}\right)}
$$

A 1-second moving window evaluated mean and variance of $\mathrm{R}_{\mathrm{xz}}$ time series. If window mean of $\mathrm{R}_{\mathrm{A}, \mathrm{xz}}$ as bounded between $0.97 \mathrm{~g}$ and $1.02 \mathrm{~g}$ and the window variance below $0.1 \mathrm{~g}$. Than the data was categorized as sleep data (figure $3 b$ ). 

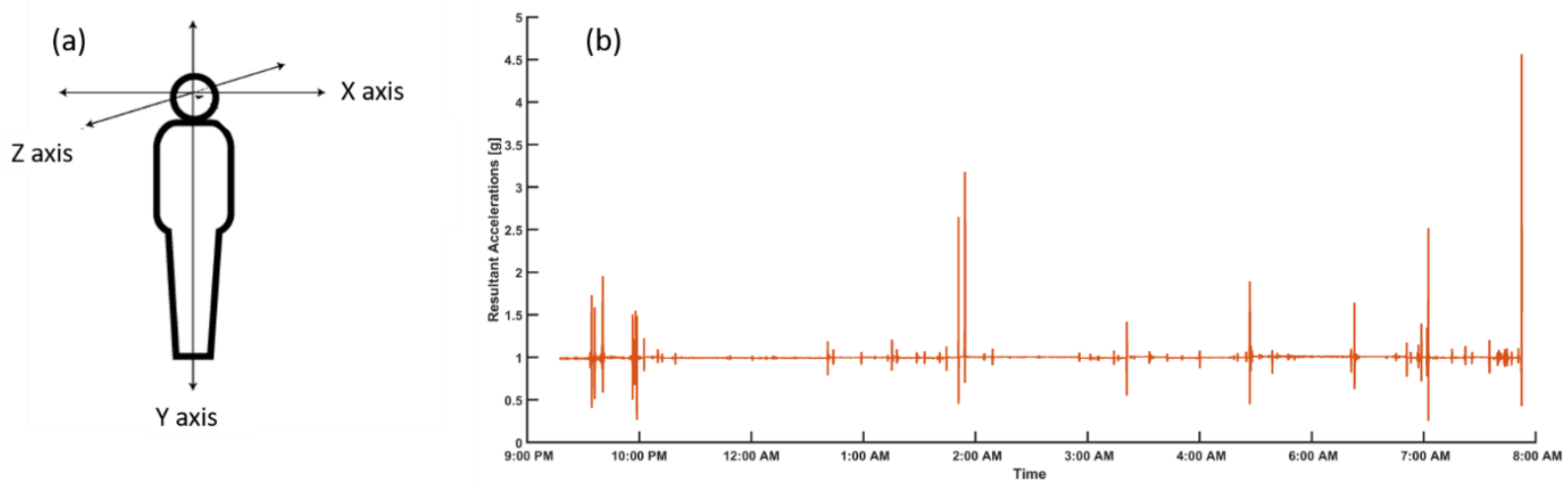

Figure 3: a) $\mathrm{X}, \mathrm{Y}$ and $\mathrm{Z}$ directions for accelerometers and gyroscopes. b) Representative data of resultant accelerations produced by sleep movements in healthy young adult

Table 2: Movement based parameters defined for a 3-Day data

\begin{tabular}{|c|c|}
\hline Parameter & Definition \\
\hline Number_of_Transitions & $\begin{array}{l}\text { Total number of transitions } \\
\text { found in one sleep dataset. }\end{array}$ \\
\hline Sleep_Hours & $\begin{array}{l}\text { Total Number of hours of } \\
\text { sleep }\end{array}$ \\
\hline Transition/Hour & $\frac{\text { Number of Transitions }}{\text { Sleep Hours }}$ \\
\hline Sleep_Phases_I & First quadrant of Sleep_hours \\
\hline Sleep_Phases_II & $\begin{array}{l}\text { Second quadrant of } \\
\text { Sleep_hours }\end{array}$ \\
\hline Sleep_Phases_III & $\begin{array}{l}\text { Third quadrant of } \\
\text { Sleep_hours }\end{array}$ \\
\hline Sleep_Phases_IV & $\begin{array}{l}\text { Fourth quadrant of } \\
\text { Sleep_hours }\end{array}$ \\
\hline Transition_Max_Acc & $\begin{array}{l}\text { Maximum of acceleration } \\
\text { value in each transition }\end{array}$ \\
\hline Transition_Min_Acc & $\begin{array}{l}\text { Minimum of acceleration } \\
\text { value in each transition }\end{array}$ \\
\hline Transition_RMS & $\begin{array}{l}\text { Root Mean Square of } \\
\text { acceleration during a } \\
\text { transition }\end{array}$ \\
\hline Transition_Range & $\begin{array}{l}\text { Differnce between max and } \\
\text { min acceleration value in } \\
\text { each transition }\end{array}$ \\
\hline
\end{tabular}




\begin{tabular}{|l|l|}
\hline Transition_Duration & $\begin{array}{l}\text { average time for each } \\
\text { transition }\end{array}$ \\
\hline
\end{tabular}

Wavelet based Frequency Analysis: For frequency analysis, Fourier transforms are first choice to determine frequency content of a signal. However, frequency characteristics of inertial sensor signals change over time and Fourier transform does not offer temporal localization. Wavelet transform are more promising in both the time and frequency domain information of the signal.

Creation of a Morlet Wavelet: In order to make a Morlet wavelet, we can create a sine wave and a Gaussian wave and multiply them point by point. Both sine wave and the Gaussian wave must have same number of time points and same sampling rate. The frequency of the wavelet is the frequency of the sine wave. The frequency of Morlet wavelet (center frequency) is actually a band of frequencies.

The Gaussian window (bell-shaped or normal curve) is expressed as:

$$
\text { Gaussian Window }=a e^{-(t)^{2} /\left(2 s^{2}\right)}
$$

Where the variable a is amplitude (height of the Gaussian curve), $t$ is the time, $s$ is standard deviation (width of Gaussian curve). The standard deviation s is defined as:

$$
s=\frac{n}{2 \pi f}
$$

Where $\mathrm{f}$ is frequency (in Hertz), $\mathrm{n}$ refers to number of wavelet cycles. Wavelet cycles ( $\mathrm{n}$ ) defines a trade-off between temporal precision of a signal to its frequency precision. Although a realvalued Morlet wavelet is created by multiplying a since wave by a Gaussian; for wavelet-based time-frequency analysis we utilize a complex Morlet wavelet $(\mathrm{cmw})$, which is created by multiplying complex sine wave with a Gaussian wave.

Wavelet-based signal analysis in time and frequency domain: In wavelet-based signal processing, the sampling rate of wavelet is set to be equal to the sampling rate of the inertial sensor signals $(\mathrm{f}=100 \mathrm{~Hz})$. The wavelet time was chosen as $4 \mathrm{~s}$ and the cycles of the gaussian window are set to be from 12 to 14 , which is relatively large since we are also interested in the frequency domain information. The wavelet function can be expressed as:

$$
\text { wavelet }=e^{i 2 \pi f * t} * e^{-\frac{t^{2}}{2 *\left(\frac{c y c l e}{2 \pi f}\right)^{2}}}
$$

Wavelet based time-frequency analysis can be conducted by time domain convolution between signal and wavelets. Here we performed frequency domain convolution since it is faster for long data sets. In order to perform a frequency domain convolution, firstly FFT was performed on signal and wavelets. This was followed by frequency domain multiplication of signal FFT and wavelet FFT. This convoluted signal in frequency domain provides complete information about 
frequency at various temporal localizations. Inverse-FFT can be performed to get convoluted signal in time domain (figure 4).

Frequency-Domain Convolution

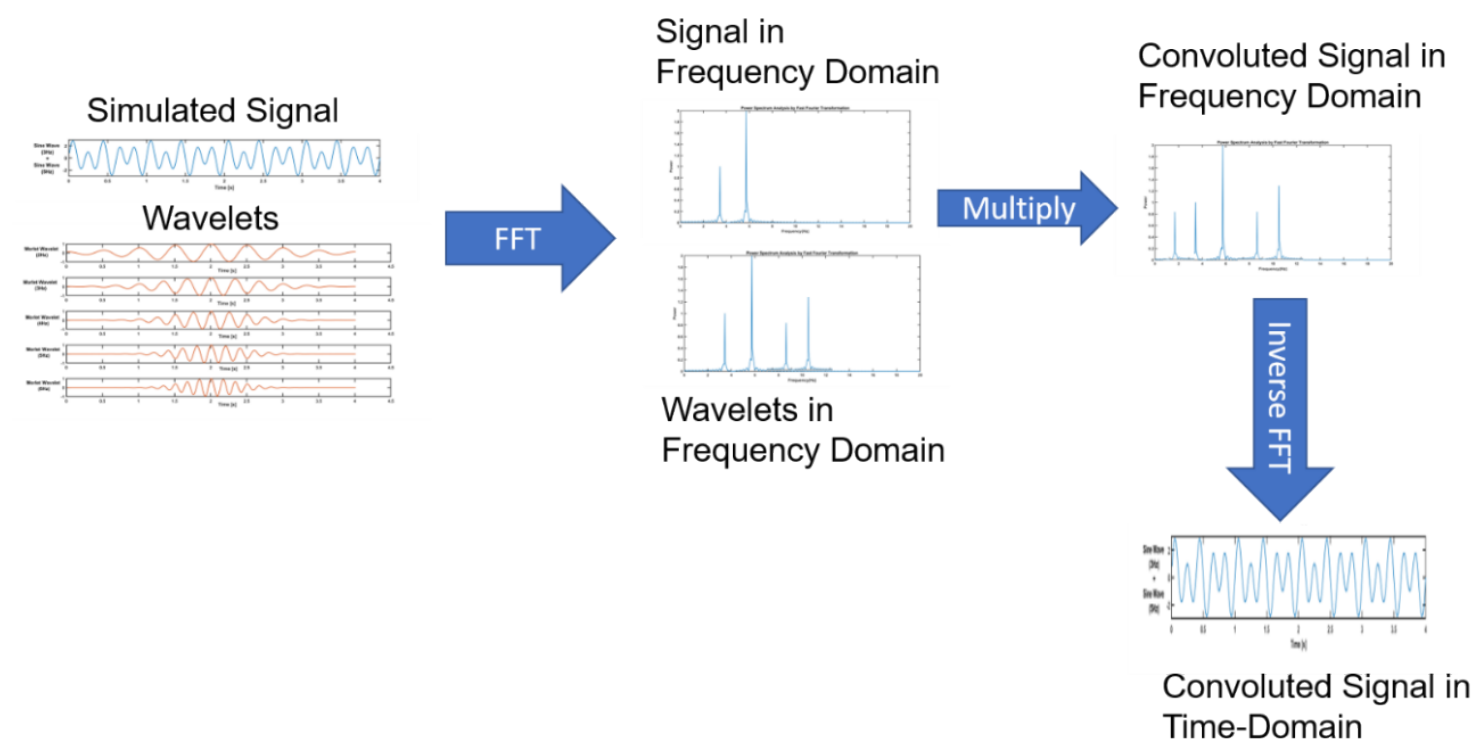

Figure 4: Illustration of the convolution theorem and frequency-domain multiplication.

The power of signal at particular frequency is a function of its amplitude:

$$
\text { power }=\frac{\text { amplitude }}{2}
$$

Morlet wavelet transform based convolution method is capable of analyzing the acceleration, angular velocity signals for longitudinal data. For wavelet-based time-frequency analysis of longitudinal inertial sensor data we have sub-categorized movements into 3 frequency bands where most of daily activity movements occur. Each frequency band is subdivided into a total of 5 frequencies. Band 1: [0.1 0.2 0.3 0.4 0.5] Hz; Band 2: [0.8 1.1 1.4 1.7 2] Hz; Band 3: [4 6810 12] Hz. Wavelets within the band frequencies were convoluted with the inertial sensor signals to extract accurate time and related frequency of movement throughout the day. Gaussian wavelet with these frequencies $(0.8 \mathrm{~Hz}$ and $1.1 \mathrm{~Hz}$ shown in figures 5 and 6$)$ to the signal to extract corresponding frequencies' behaviors.

\section{Results}

Figures 5 and 6 show an example of outcome for 24-hour (day) frequency analysis. The yellow line is the average power for each group (10 participants in each group). 

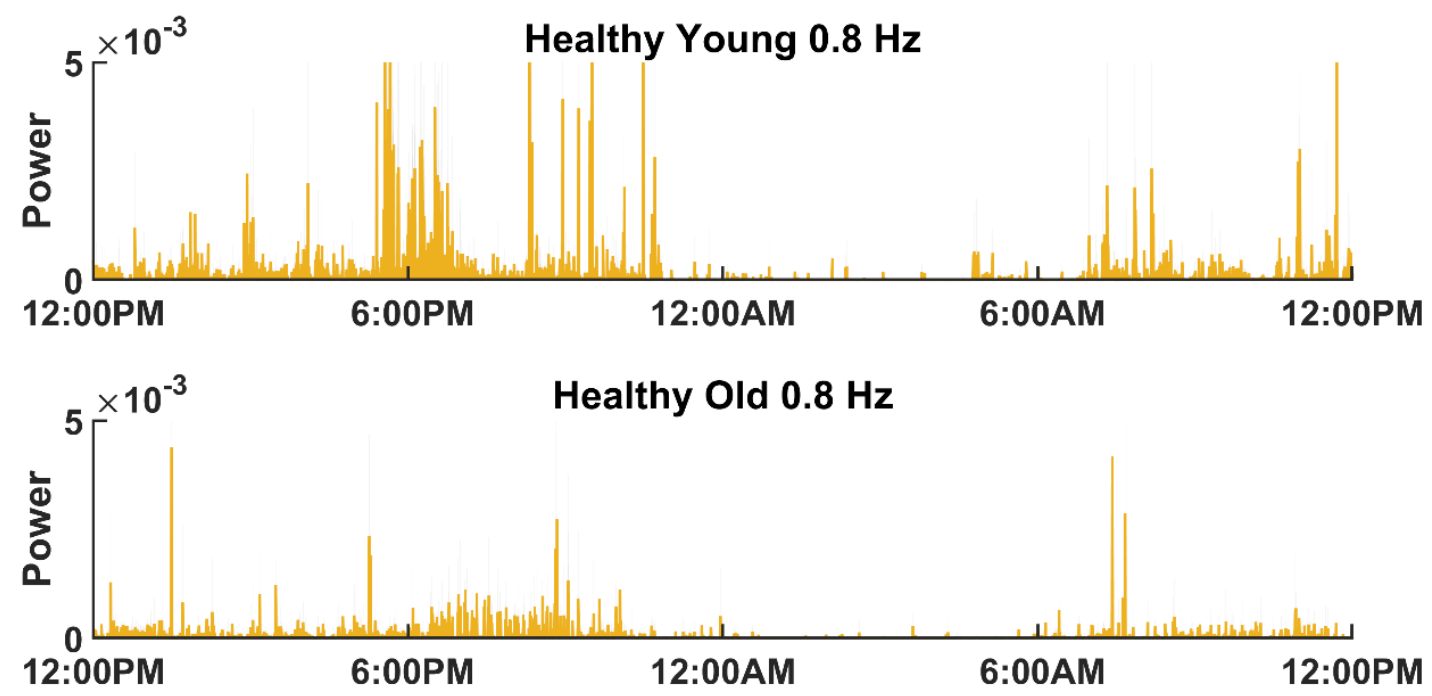

Figure 5 Average power and std for Healthy Young and Healthy Old group at $0.8 \mathrm{~Hz}$ movements throughout 24-hour day
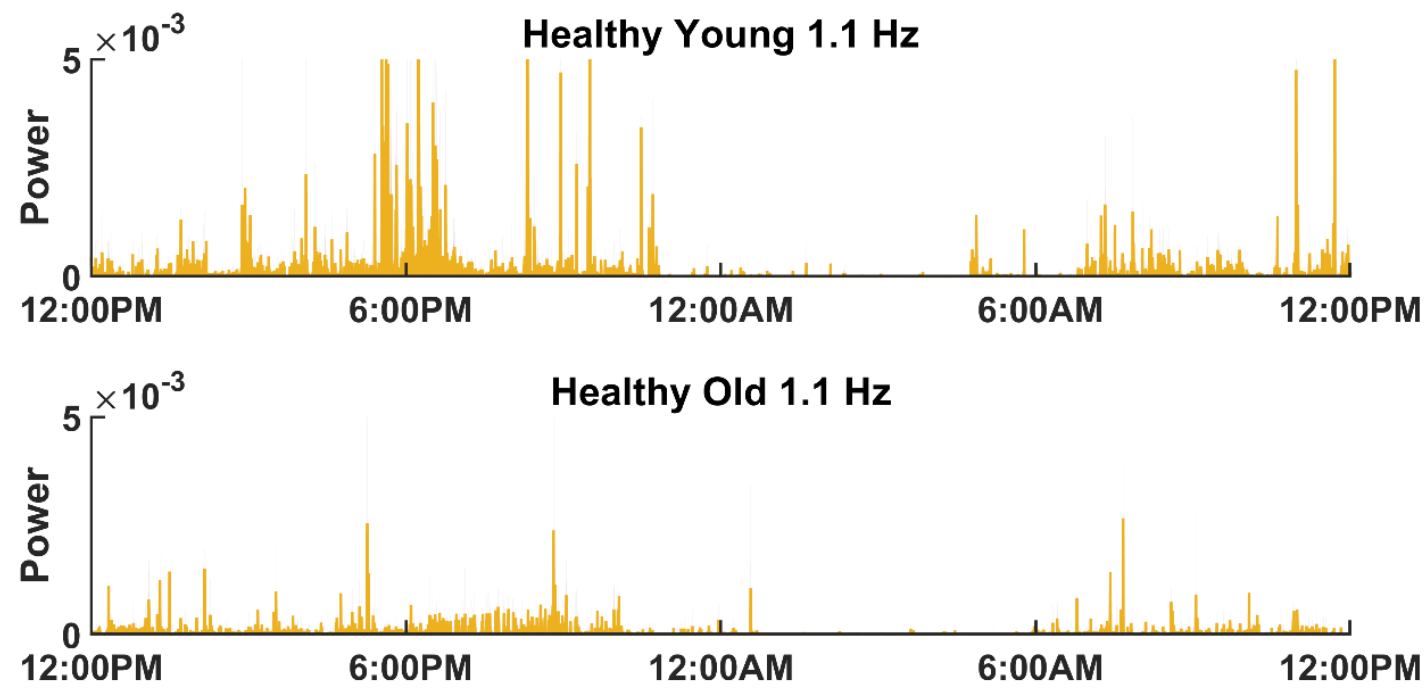

Figure 6 Average power and SD for Healthy Young and Healthy Old group at $1.1 \mathrm{~Hz}$ movements throughout 24-hour day

All movements were divided into four time zones of the 24-hour day as shown in table 3 . Table 4 and table 5 shows how much time and amplitude of low, medium and large movements were performed in the four different time zones. 
Table 3: The movements during the day were divided into 4 time-zones.

\begin{tabular}{|l|l|}
\hline Time Zones & Time of Day \\
\hline $\begin{array}{l}\text { Time Zone 1 } \\
(\mathrm{TZ1})\end{array}$ & 12AM $<$ Time $\leq 6$ \\
\hline $\begin{array}{l}\text { Time Zone 2 } \\
(\mathrm{TZ2})\end{array}$ & $\begin{array}{l}\text { 6 AM }<\text { Time } \leq 12 \\
\text { PM }\end{array}$ \\
\hline $\begin{array}{l}\text { Time Zone } 3 \\
\text { (TZ3) }\end{array}$ & $12 \mathrm{PM}<$ Time $\leq 6 \mathrm{PM}$ \\
\hline $\begin{array}{l}\text { Time Zone 4 } \\
(\mathrm{TZ4})\end{array}$ & $\begin{array}{l}\text { 6 PM }<\text { Time } \leq 12 \\
\text { PM }\end{array}$ \\
\hline
\end{tabular}

Table 4: Time in hours spent during low, medium and high magnitudes of activities throughout 4 time zones in a day

\begin{tabular}{|l|l|l|l|}
\hline Amplitude & $\begin{array}{l}\text { Time } \\
\text { Zone }\end{array}$ & $\begin{array}{l}\text { Healthy } \\
\text { Old }\end{array}$ & $\begin{array}{l}\text { Healthy } \\
\text { Young }\end{array}$ \\
\hline Low & TZ1 & $5.46 \pm 0.46$ & $5.28 \pm 0.38$ \\
\hline & TZ2 & $5.90 \pm 0.05$ & $5.48 \pm 0.39$ \\
\hline & TZ3 & $5.99 \pm 0.02$ & $5.93 \pm 0.14$ \\
\hline & TZ4 & $5.64 \pm 0.16$ & $5.57 \pm 0.32$ \\
\hline Medium & TZ1 & $0.44 \pm 0.32$ & $0.61 \pm 0.34$ \\
\hline & TZ2 & $0.10 \pm 0.05$ & $0.46 \pm 0.33$ \\
\hline & TZ3 & $0.01 \pm 0.02$ & $0.06 \pm 0.12$ \\
\hline & TZ4 & $0.36 \pm 0.15$ & $0.36 \pm 0.26$ \\
\hline High & TZ1 & $0.10 \pm 0.21$ & $0.11 \pm 0.13$ \\
\hline & TZ2 & $0.00 \pm 0.00$ & $0.06 \pm 0.07$ \\
\hline & TZ3 & $0.00 \pm 0.00$ & $0.01 \pm 0.02$ \\
\hline & TZ4 & $0.01 \pm 0.01$ & $0.07 \pm 0.11$ \\
\hline
\end{tabular}

Table 5: Amplitudes in absolute value spent during low, medium and high magnitudes of activities throughout 4 time zones in a day

\begin{tabular}{|l|l|l|l|}
\hline Amplitude & $\begin{array}{l}\text { Time } \\
\text { Zone }\end{array}$ & Healthy Old & Healthy Young \\
\hline Low & TZ1 & $43121.1 \pm 9533.8$ & $38205.5 \pm 13265.1$ \\
\hline & TZ2 & $30998.0 \pm 18543.8$ & $39151.4 \pm 11008.7$ \\
\hline & TZ3 & $34949.1 \pm 18916.2$ & $26029.7 \pm 10063.0$ \\
\hline & TZ4 & $32134.9 \pm 10440.0$ & $32455.5 \pm 9821.3$ \\
\hline Medium & TZ1 & $22341.3 \pm 21854.6$ & $31838.8 \pm 19234.3$ \\
\hline & TZ2 & $2928.8 \pm 1771.0$ & $20570.5 \pm 16554.4$ \\
\hline & TZ3 & $412.0 \pm 589.7$ & $3163.3 \pm 7344.8$ \\
\hline & TZ4 & $13450.2 \pm 6808.9$ & $17670.7 \pm 13747.7$ \\
\hline
\end{tabular}




\begin{tabular}{|l|l|l|l|}
\hline High & TZ1 & $8507.0 \pm 17789.1$ & $15143.0 \pm 20982.4$ \\
\hline & TZ2 & $121.1 \pm 136.1$ & $8256.8 \pm 10050.6$ \\
\hline & TZ3 & $8.5 \pm 14.8$ & $898.2 \pm 2348.5$ \\
\hline & TZ4 & $807.9 \pm 12.5$ & $10774.1 \pm 23935.1$ \\
\hline
\end{tabular}

A 2(age) X 4(TZ) X 3(low, medium, high magnitude) mixed design MANOVA was conducted with number of transitions and time of transition data.

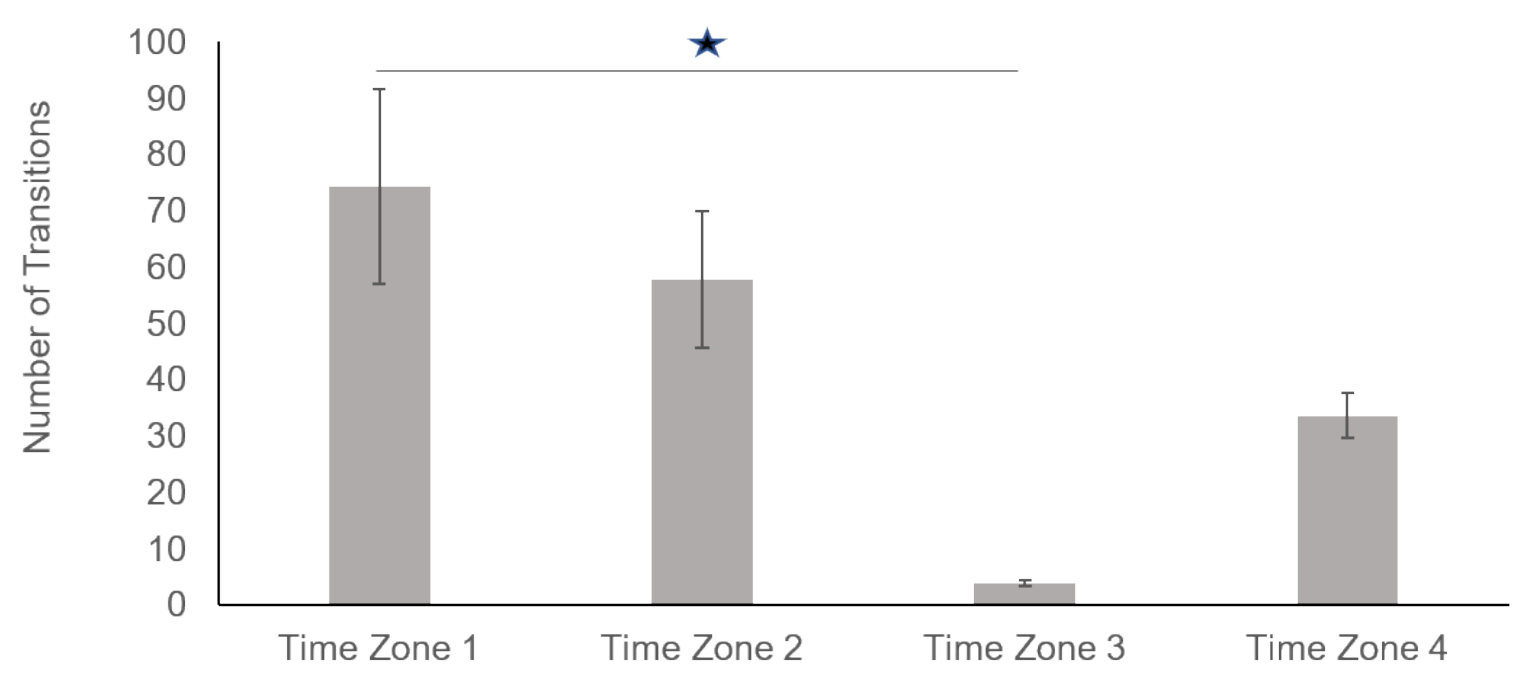

Figure 7: Number of transitions performed in the four time zones.

It was found that both young and older adults performed significantly more activities during Time Zone 1 with respect to Time zone 3 (figure 7). It was also found that both young and older participants performed significantly more low intensity transitions than medium and high amplitude transitions (figure 8). 


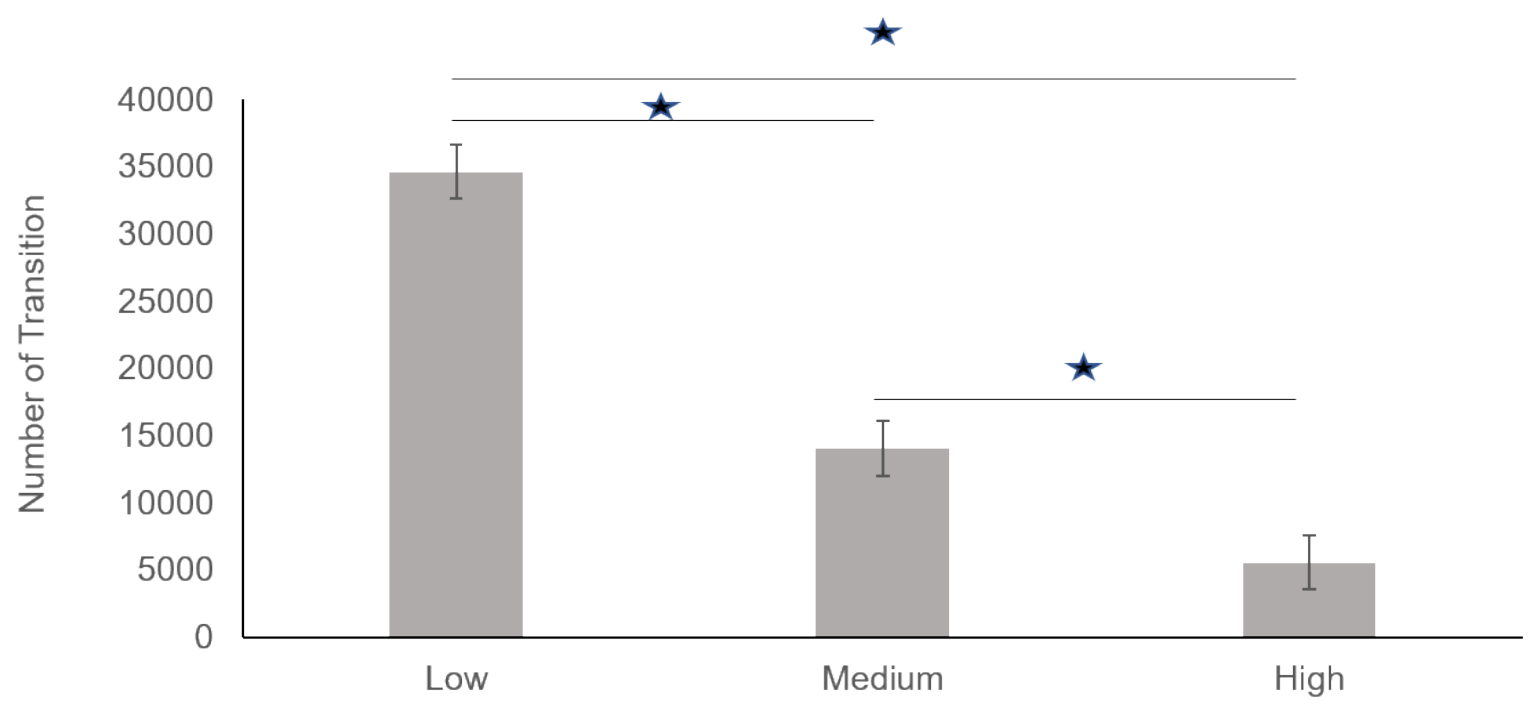

Absolute Acceleration Magnitude

Figure 8: Number of transitions performed at low, medium and high amplitude.

We found that healthy younger group performed significantly higher number of movements at $1.1 \mathrm{~Hz}$ (figure 9). 


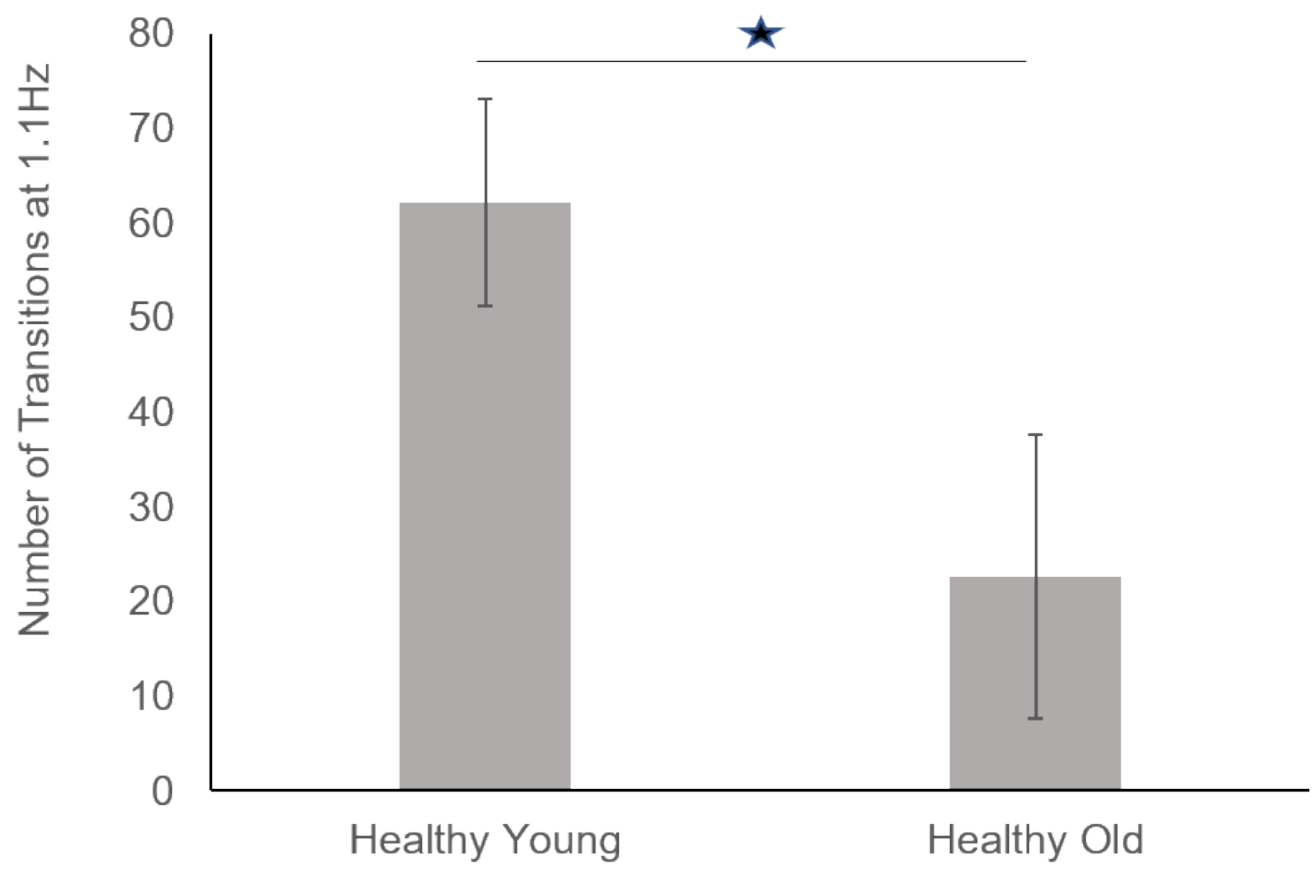

Figure 9: Number of transitions performed at $1.1 \mathrm{~Hz}$ by healthy young and older adults

Sleep Analysis: Sleep was divided into 4 equal phases. It was found that younger participants produced significantly less transitions during the middle phases (phase 2 and phase 3 ) (figure 10). But no significant differences were found in phases among older participants. We also found that young adults produced significantly more accelerations in magnitude than the older adults (figure 11). 
(a)

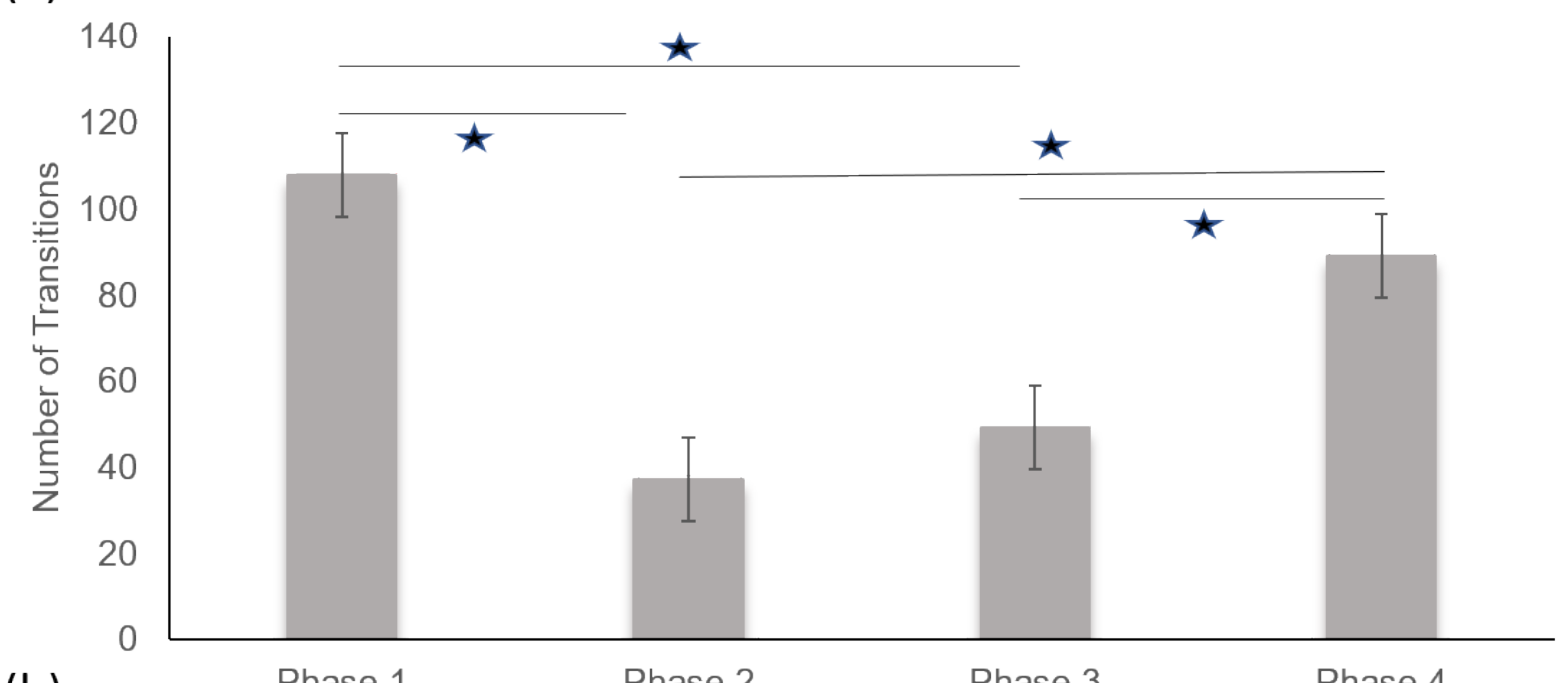
(b)
Phase 1
Phase 2
Phase 3
Phase 4

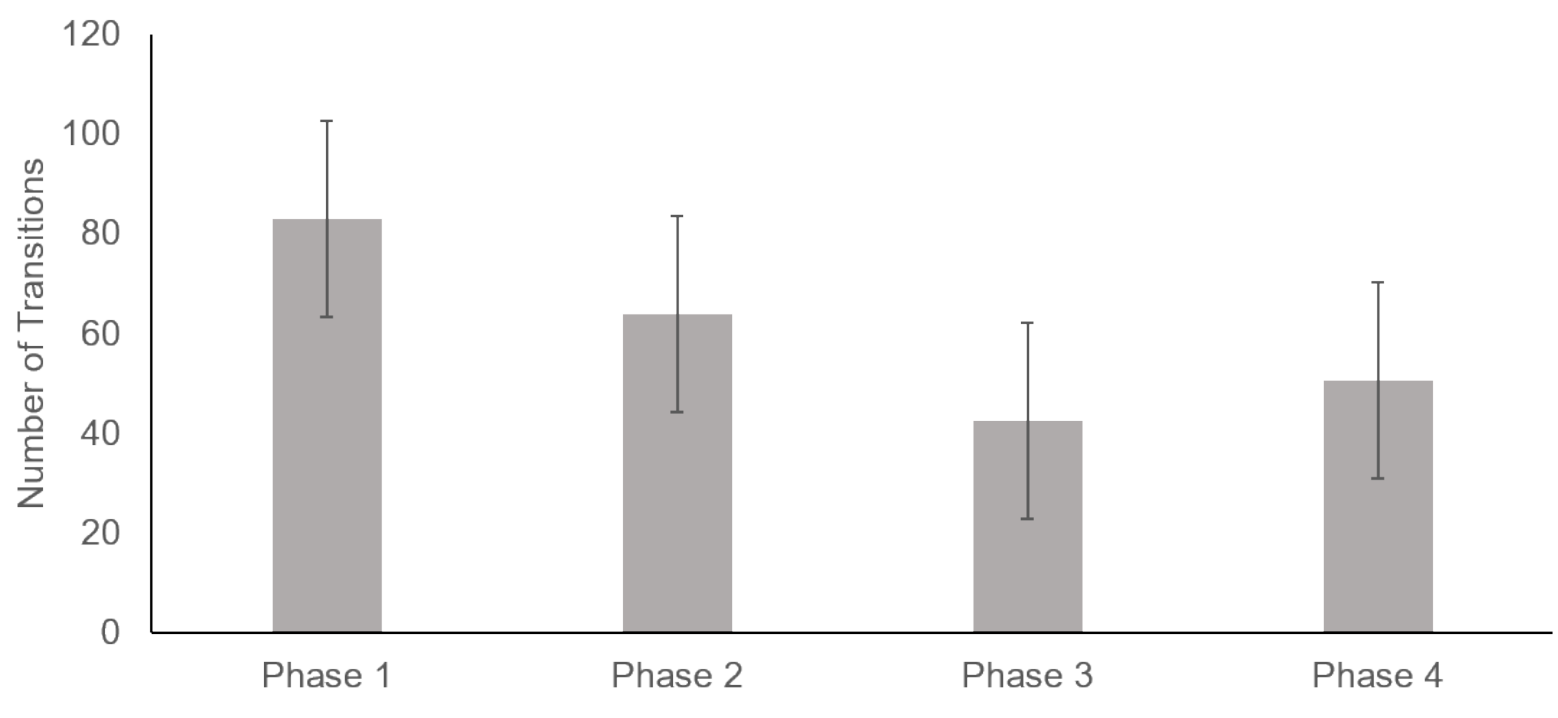

Figure 10: (a) Healthy young participants with significantly lower number of transitions in phase 2 and phase 3. (b) Healthy Older participants and number of transitions produced during all four phases of sleep 


\section{Maximum Transition Acceleration}

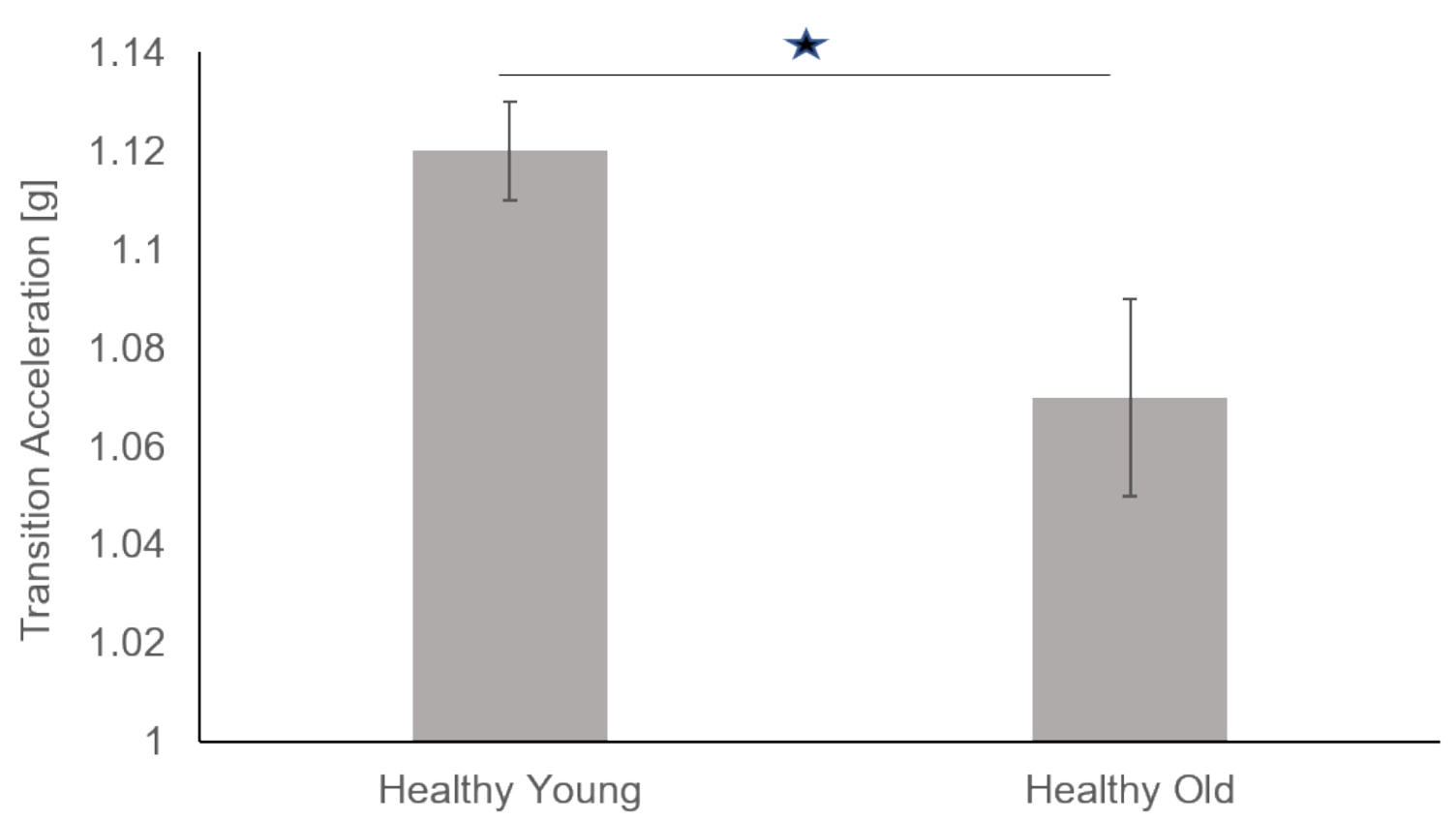

Figure 11: Maximum Accelerations produced during transitions in young and older adults

\section{Discussion}

One of the biggest challenges for our society is to serve fast growing older population. There is a growing concern on how to help older adults to live independently and in good health and with better quality of life. The longitudinal monitoring utilizing inertial sensors has an enormous potential to achieve this goal. However, today there is a knowledge gap about how the technology could be brought to daily clinical use. One explanation for this gap is the huge biomedical movement signals from the sensors. There is not enough information available how these signals can provide clinically relevant information. Research in this area has to be planned and performed in close cooperation of bioengineers with medical doctors or care givers such to develop simple, reliable, and practically useful alert and diagnostic system. The goal of this work was to investigate novel methods to understand human movements during performance of activities of daily living in healthy young and older adults. A second objective was to be able to characterize these movements from lower trunk acceleration and angular velocity signals into parameters which can be helpful to assess and compare human mobility. Our previous findings (Lockhart et al., 2013; Soangra \& Lockhart, 2012) suggest that a single wearable sensor can be helpful in distinguishing mobility among healthy young and older adults. Similarly, another research has showed that a single waist worn sensor can detect walking with high accuracy (accuracy $>90 \%$ ) (Oshima et al., 2010; Sekine, Tamura, Togawa, \& Fukui, 2000). 
In this study, subjects wore one wearable device at low-back and did not experience any interference or inconvenience when performing their daily activities. We found that both young and older participants performed significantly more activities during early morning (TZ1) (figure 7). This could be a result of daily busy working schedule of healthy young and older population. We found that very less activities were performed during late evening (TZ3) (figure 7). This could have been influenced by resting in home environments during 6PM to $12 \mathrm{PM}$. It is also found that both young and older adults perform significantly more low magnitude acceleration movements than medium and high acceleration movements (figure 8). The amplitude-wise movement classification in the four different time zones (table 3) are shown in table 5. It was found that both healthy young and old spent more than 5 hours in performing low magnitude activities. Whereas younger adults performed more of medium magnitude activities (table 4). Using time-frequency analysis we found that younger adults performed significantly more movements at the frequency of $1.1 \mathrm{~Hz}$ (figure 9). This can also be justified by walking, since walking occurs around $1 \mathrm{~Hz}$ and younger walk more than older counterparts. Activity monitors have been used previously to estimate sleep duration in studies where polysomnography (PSG) would be intrusive, burdensome or expensive(Mullaney, Kripke, \& Messin, 1980). Activity monitors have utilized threshold-motion detectors to estimate sleep with good concordance with PSG (Sadeh, Hauri, Kripke, \& Lavie, 1995). In this study we have examined nocturnal sleep period with magnitude and number of transitions. We have compared postural transitions made in the bed in four different phases of sleep between healthy young and older adults. We found younger adults make significantly less transitions in phase 2 and phase 3 of their sleep time. This may be a signature of a deep sleep at night and a precursor to good overall health. We also found that younger adults produced transitions of significantly greater accelerations throughout night when compared to older adults (figure 11). The experimental protocol adopted in this study proved to be suitable to investigate the differences in activity and sleep in healthy young and older adults. The new methods in this study using wearable sensor could be a promising technique for objective, unobtrusive and continuous evaluation of physical functioning or effects of interventions (e.g. medication, physiotherapy). Future research with the long-term quantitative assessment of movements can be directed to determine whether there are inter- and intrapersonal differences (i.e., between persons and over time) in movement characteristics. These inter and intra individual variabilities in movement frequencies can be indicators of good health. Longitudinal assessments will help improve clinical results and their understanding how the progression of diseases or the recovery from a disease influence the movement characteristics of the patients.

\section{Conclusion}

In the $21^{\text {st }}$ century, there is growing interest in having objective assessment of health-related outcomes using technology-based wearable devices that can provide unbiased measurements of human mobility related health assessments which can be used in clinical practice and scientific research. Wearable sensor monitor system is a practical and valuable tool for objective, continuous evaluation of human movements involved in activities of daily living and sleep in healthy individuals and patients. The study was designed to evaluate temporal and frequency- 
based parameters which could help investigate longitudinal data of 3-days. This study showed that the presented novel algorithms can help understand mobility deficits and sleep abnormalities over longitudinal inertial sensor data from low-back accelerations and angular velocities, which provides opportunities to extract a range of mobility parameters from wearable sensors. This research has potential to monitor disease progression and its effects on human movement and sleep. It also has potential to assess treatment effectiveness accurately on activities of daily living. In future, longitudinal human movement data and sleep transition data from wearable sensors combined with artificial intelligence algorithms can be helpful to diagnose abnormality and disease progression in the healthcare domain.

\section{References}

Allet, L., Knols, R. H., Shirato, K., \& de Bruin, E. D. (2010). Wearable systems for monitoring mobility-related activities in chronic disease: a systematic review. Sensors (Basel), 10(10), 9026-9052. doi:10.3390/s101009026

Aminian, K., \& Najafi, B. (2004). Capturing human motion using body-fixed sensors: outdoor measurement and clinical applications. Computer Animation and Virtual Worlds, 15(2), 79-94. doi:10.1002/cav.2

Brandes, M., Zijlstra, W., Heikens, S., van Lummel, R., \& Rosenbaum, D. (2006). Accelerometry based assessment of gait parameters in children. Gait Posture, 24(4), 482486. doi:10.1016/j.gaitpost.2005.12.006

Bruce, B., \& Fries, J. F. (2003). The Stanford Health Assessment Questionnaire: a review of its history, issues, progress, and documentation. J Rheumatol, 30(1), 167-178.

Bureau, U. C. (2018). Facts for features: Older Americans Month: May 2017. Retrieved from https:/www.census.gov/newsroom/facts-for-features/2017/cb17-ff08.html

Carlsson, G., Haak, M., Nygren, C., \& Iwarsson, S. (2012). Self-reported versus professionally assessed functional limitations in community-dwelling very old individuals. Int J Rehabil Res, 35(4), 299-304. doi:10.1097/MRR.0b013e3283544d07

Centers for Disease Control and Prevention. (2015). Retrieved from https://www.cdc.gov/physicalactivity/basics/index.htm?CDC_AA refVal=https $\% 3 \mathrm{~A} \% 2 \mathrm{~F}$ $\% 2 \mathrm{Fwww} . c d c . g o v \% 2 \mathrm{Fphysicalactivity} \% 2 \mathrm{Fbasics} \% 2 \mathrm{Fpa}$-health $\% 2 \mathrm{Findex} . \mathrm{htm}$.

Czeisler, C. A., Dumont, M., Duffy, J. F., Steinberg, J. D., Richardson, G. S., Brown, E. N., ... . Ronda, J. M. (1992). Association of sleep-wake habits in older people with changes in output of circadian pacemaker. Lancet, 340(8825), 933-936.

Driscoll, H. C., Serody, L., Patrick, S., Maurer, J., Bensasi, S., Houck, P. R., . . Reynolds, C. F., 3rd. (2008). Sleeping well, aging well: a descriptive and cross-sectional study of sleep in "successful agers" 75 and older. Am J Geriatr Psychiatry, 16(1), 74-82. doi:10.1097/JGP.0b013e3181557b69

Gloeckl, R., Damisch, T., Prinzen, J., van Lummel, R., Pengel, E., Schoenheit-Kenn, U., \& Kenn, K. (2015). Validation of an activity monitor during sleep in patients with chronic respiratory disorders. Respir Med, 109(2), 286-288. doi:10.1016/j.rmed.2014.12.017

Gokalp, H., \& Clarke, M. (2013). Monitoring activities of daily living of the elderly and the potential for its use in telecare and telehealth: a review. Telemed J E Health, 19(12), 910923. doi:10.1089/tmj.2013.0109

Liu, J., Sohn, J., \& Kim, S. (2017). Classification of Daily Activities for the Elderly Using Wearable Sensors. J Healthc Eng, 2017, 8934816. doi:10.1155/2017/8934816 
Lockhart, T. E., Soangra, R., Zhang, J., \& Wu, X. (2013). Wavelet based automated postural event detection and activity classification with single imu - biomed 2013. Biomed Sci Instrum, 49, 224-233.

Logan, S. L., Gottlieb, B. H., Maitland, S. B., Meegan, D., \& Spriet, L. L. (2013). The Physical Activity Scale for the Elderly (PASE) questionnaire; does it predict physical health? Int J Environ Res Public Health, 10(9), 3967-3986. doi:10.3390/ijerph10093967

Mathie, M. J., Celler, B. G., Lovell, N. H., \& Coster, A. C. (2004). Classification of basic daily movements using a triaxial accelerometer. Med Biol Eng Comput, 42(5), 679-687.

Mullaney, D. J., Kripke, D. F., \& Messin, S. (1980). Wrist-actigraphic estimation of sleep time. Sleep, 3(1), 83-92.

Oshima, Y., Kawaguchi, K., Tanaka, S., Ohkawara, K., Hikihara, Y., Ishikawa-Takata, K., \& Tabata, I. (2010). Classifying household and locomotive activities using a triaxial accelerometer. Gait \& Posture, 31(3), 370-374. doi:10.1016/j.gaitpost.2010.01.005

Potter, G. D. M., Skene, D. J., Arendt, J., Cade, J. E., Grant, P. J., \& Hardie, L. J. (2016). Circadian Rhythm and Sleep Disruption: Causes, Metabolic Consequences, and Countermeasures. Endocrine Reviews, 37(6), 584-608. doi:10.1210/er.2016-1083

Sadeh, A., Hauri, P. J., Kripke, D. F., \& Lavie, P. (1995). The role of actigraphy in the evaluation of sleep disorders. Sleep, 18(4), 288-302.

Sekine, M., Tamura, T., Togawa, T., \& Fukui, Y. (2000). Classification of waist-acceleration signals in a continuous walking record. Medical Engineering \& Physics, 22(4), 285-291. doi:10.1016/s1350-4533(00)00041-2

Soangra, R., \& Lockhart, T. E. (2012). A comparative study for performance evaluation of sit-tostand task with body worn sensor and existing laboratory methods. Biomed Sci Instrum, $48,407-414$.

Soangra, R., Lockhart, T. E., Lach, J., \& Abdel-Rahman, E. M. (2013). Effects of hemodialysis therapy on sit-to-walk characteristics in end stage renal disease patients. Ann Biomed Eng, 41(4), 795-805. doi:10.1007/s10439-012-0701-6

Zijlstra, W. (2004). Assessment of spatio-temporal parameters during unconstrained walking. Eur J Appl Physiol, 92(1-2), 39-44. doi:10.1007/s00421-004-1041-5

Zijlstra, W., \& Hof, A. L. (2003). Assessment of spatio-temporal gait parameters from trunk accelerations during human walking. Gait Posture, 18(2), 1-10. 